\title{
THE LARGE-TIME SOLUTION OF A NONLINEAR FOURTH-ORDER EQUATION INITIAL-VALUE PROBLEM I. INITIAL DATA WITH A DISCONTINUOUS EXPANSIVE STEP
}

\author{
J. A. LEACH ${ }^{\otimes 1}$ and ANDREW P. BASSOM ${ }^{2}$
}

(Received 29 November, 2008; revised 25 November, 2009)

\begin{abstract}
In this paper we consider an initial-value problem for the nonlinear fourth-order partial differential equation $u_{t}+u u_{x}+\gamma u_{x x x x}=0,-\infty<x<\infty, t>0$, where $x$ and $t$ represent dimensionless distance and time respectively and $\gamma$ is a negative constant. In particular, we consider the case when the initial data has a discontinuous expansive step so that $u(x, 0)=u_{0}(>0)$ for $x \geq 0$ and $u(x, 0)=0$ for $x<0$. The method of matched asymptotic expansions is used to obtain the large-time asymptotic structure of the solution to this problem which exhibits the formation of an expansion wave. Whilst most physical applications of this type of equation have $\gamma>0$, our calculations show how it is possible to infer the large-time structure of a whole family of solutions for a range of related equations.
\end{abstract}

2000 Mathematics subject classification: primary 35B40; secondary 34E10.

Keywords and phrases: partial differential equation, asymptotic analysis.

\section{Introduction}

In this article we are concerned with an initial-value problem for the fourth-order evolution equation

$$
u_{t}+u u_{x}+\gamma u_{x x x x}=0, \quad-\infty<x<\infty, t>0,
$$

where $\gamma<0$ is a constant, subject to the initial conditions

$$
u(x, 0)= \begin{cases}u_{0}, & x \geq 0 \\ 0, & x<0\end{cases}
$$

\footnotetext{
${ }^{1}$ School of Mathematics, University of Birmingham, Edgbaston, Birmingham B15 2TT, UK; e-mail: leachja@for.mat.bham.ac.uk.

${ }^{2}$ School of Mathematics and Statistics, University of Western Australia, Crawley 6009, Australia; e-mail: bassom@maths.uwa.edu.au.

(C) Australian Mathematical Society 2010, Serial-fee code 1446-1811/2010 \$16.00
} 
and boundary conditions

$$
u(x, t) \rightarrow\left\{\begin{array}{ll}
u_{0}, & x \rightarrow \infty, \\
0, & x \rightarrow-\infty,
\end{array} \quad t \geq 0\right.
$$

The initial distribution (1.2) is a discontinuous expansive step with $u_{0}>0$ and in what follows we shall refer to (1.1)-(1.3) as IVP.

Equation (1.1) can be regarded as a fourth-order viscosity extension of the firstorder conservation law

$$
u_{t}+u u_{x}=0,
$$

which is readily recognized as the inviscid Burgers' equation. Then the solution of the Riemann problem IVP takes the form of a shock wave (see [3] and the references therein). Our objective in this work is to examine the large-time structure of the solution of IVP when $\gamma<0$. In this case the discontinuous initial data collapses into the smooth expansion (rarefaction) wave for $t>0$. The piecewise initial form of the expansion wave is smoothed by the fourth-order derivative.

It is clear that our problem is technically ill-posed, in the sense that the solution $u=0$, for $x<0$ when $t=0$, is linearly unstable. However, our intention here is to illustrate how the discontinuous jump in the initial profile at $x=0$ develops as $t \rightarrow \infty$. For this purpose, the exact form of the initial profile in $x<0$ is only of secondary importance, and to simplify the presentation the assumption of $u(x, 0)=0$ for $x<0$ has been made. Once the main ingredients of the solution procedure have been appreciated, it is quite straightforward to adapt the arguments should some other initial (linearly stable) profile be appropriate.

Although second-order diffusion occurs frequently within many problems in applied mathematics, it has been recognized that fourth-order terms do appear in several applications. Space precludes anything more than a cursory overview of some of the instances in which fourth-order diffusion arises, but we mention the studies of Broadbridge and Tritscher [1] who derived equations for the thermal grooving of metals and the work of Kyrychko et al. [5] concerned with the existence of travelling waves in higher-order biological problems. Higher-order diffusion methods are also being increasingly used in modern computational fluid dynamics; see, for example, [2] and the many references within. Our contribution here does not focus on such complicated systems but rather is a first step in the analysis of Equation (1.1) that promises to form the basis for addressing the generalized Kuramoto-Sivashinsky equation

$$
u_{t}+u u_{x}+\alpha u_{x x}+\beta u_{x x x}+\gamma u_{x x x x}=0,
$$

where $\alpha$ and $\beta$ are constants.

Here the aim is to use the method of matched asymptotic expansions to develop the large-time asymptotic structure of the solution to IVP when the initial data (1.2) is a discontinuous expansive step. The monograph [7] shows that the evolution of the solution is sensitive to the form of the initial condition and whether this profile 
is a continuous function of $x$ or contains jumps. Hence our choice here is to focus on the step-problem IVP and defer the consideration of the continuous initial profile to future work. Throughout we use the nomenclature of the theory of matched asymptotic expansions, as given in Van Dyke [10] (see also Hinch [4], Lagerstrom [6] or Nayfeh [9] for an introduction to the theory of matched asymptotic expansions). We shall see how it is possible to infer the $t \gg 1$ structure of the solution with minimal computational effort. At face value, it is somewhat of a surprise that this can be done; after all, it would be expected that part of the calculation to derive the large-time form for all $x$ would require consideration of the solution in the parameter space where both $x$ and $t$ are $O(1)$ and properties of the solution in this regime are only amenable to numerical simulation.

We demonstrate how matched asymptotics can neatly side-step this difficulty. First, we can use the initial data to calculate the small-time structure of the solution for all $x$. Then, rather than develop the form of the solution where $x=O(1)$, we instead concentrate on the evolution of the solution in the far field $|x| \rightarrow \infty$ for $t \geq O(1)$. This large- $|x|$ structure for $t \gg 1$ can in turn be used to deduce the long-time form of solution for all $x$. This strategy is not new and was originally developed in the context of reaction-diffusion equations (see [7]).

We shall show in Section 2 how the $t \gg 1$ structure assumes the form of an expansion wave. Specifically

$$
u(x, t) \sim \frac{x}{t}, \quad 0<x<u_{0} t \quad \text { as } t \rightarrow \infty
$$

and outside this range $u(x, t)$ differs by only exponentially small quantities from the respective far-field behaviours as $x \rightarrow \pm \infty$.

We note in passing that Equation (1.1) with $\gamma<0$ can be reduced via the transformation

$$
u=(-\gamma)^{1 / 4} \bar{u}, \quad x=(-\gamma)^{1 / 4} \bar{x},
$$

to the parameterless form

$$
\bar{u}_{t}+\bar{u} \bar{u}_{\bar{x}}-\bar{u}_{\bar{x} \bar{x} \bar{x} \bar{x}}=0 .
$$

However, as our future aim is to analyse the generalized Kuramoto-Sivashinsky equation (1.4) it will be instructive in this paper to retain the parameter $\gamma$, particularly since it adds no further complication, and will continue to work with Equation (1.1) in what follows.

\section{The asymptotic solution as $t \rightarrow \infty$}

In this section we develop the asymptotic structure of IVP as $t \rightarrow \infty$ following the approach outlined above. That is, the calculation proceeds through three key steps. First we develop the small-time form of the solution, then consider the evolution of this solution at $|x| \gg 1$ through to large times, and finally close the work by deducing the large-time form for all $x$. We tackle the first stage next. 
2.1. Small-time solution The examination of the initial data (1.2) suggests that the structure of the asymptotic solution to IVP as $t \rightarrow 0$ consists of three distinct zones. Clearly there must be a rapid change in the solution around the step in the initial data at $x=0$ and this region, which we shall call Ia, is sandwiched between the two zones $x>0$ and $x<0$. In summary, then, this small-time regime $\mathbf{I}$ is comprised of the three sub-regions divided as

$$
\left.\begin{array}{lll}
\text { region Ia: } & x=o(1), & u(x, t)=O(1) \\
\text { region Ib: } & x>0, & u(x, t)=u_{0}-o(1) \\
\text { region Ic: } & x<0, & u(x, t)=o(1)
\end{array}\right\} \quad \text { as } t \rightarrow 0 \text {. }
$$

2.1.1. Region Ia: $x=o(1)$ Here we introduce the scaled coordinate $\eta=x t^{-\lambda}=$ $O(1)$ as $t \rightarrow 0$, where $\lambda>0$, and seek an expansion of the form

$$
u(\eta, t)=\bar{u}(\eta)+o(1) .
$$

On substitution of expansion (2.1) into Equation (1.1) (rewritten in terms of $\eta$ and $t$ ) we find that the leading-order balance requires $\lambda=1 / 4$. Then

$$
\bar{u}_{\eta \eta \eta \eta}-\frac{\eta}{4 \gamma} \bar{u}_{\eta}=0
$$

which must be solved subject to matching with regions Ib (as $\eta \rightarrow \infty$ ) and Ic (as $\eta \rightarrow-\infty)$ and initial condition (1.2) so that

$$
\begin{array}{ll}
\bar{u}(\eta)=u_{0}-o(1) & \text { as } \eta \rightarrow \infty, \\
\bar{u}(\eta)=o(1) & \text { as } \eta \rightarrow-\infty .
\end{array}
$$

On writing $\bar{u}(\eta)=u_{0} / 2+\hat{u}(\eta)$, Equation (2.2) becomes

$$
\hat{u}_{\eta \eta \eta \eta}-\frac{\eta}{4 \gamma} \hat{u}_{\eta}=0, \quad-\infty<\eta<\infty,
$$

and so we require the odd solution of (2.3) with

$$
\hat{u}(\eta) \rightarrow \pm \frac{u_{0}}{2} \quad \text { as } \eta \rightarrow \pm \infty
$$

this can be expressed as

$$
\hat{u}(\eta)=\int_{0}^{\eta} F(s) d s
$$

where $F(\eta)$ is a solution of the third-order linear equation

$$
F_{\eta \eta \eta}-\frac{\eta}{4 \gamma} F=0
$$

We see that $F(\eta)=F(-\eta)$ and further note that (2.6) has a unique (within a constant multiple) monotone decreasing solution on $[0, \infty)$. This Kneser-type solution 
has $F(0)=A(>0)$ and $F^{\prime \prime}(0)=0$ with $F(\eta)>0, F^{\prime}(\eta) \leq 0$ and $F^{\prime \prime}(\eta) \geq 0$ on $[0, \infty)$; here $A$ is a constant to be determined. Furthermore,

$$
F(\eta)=O\left[\eta^{-1 / 3} \exp \left(-3(-256 \gamma)^{-1 / 3} \eta^{4 / 3}\right)\right] \quad \text { as } \eta \rightarrow \infty
$$

and then the boundary conditions (2.4) require that the constant $A$ is fixed so that

$$
\int_{0}^{\infty} F(s) d s=\frac{1}{2} u_{0} .
$$

Therefore, in region Ia we have

$$
u(\eta, t)=\frac{1}{2} u_{0}+\int_{0}^{\eta} F(s) d s+o(1) \quad \text { as } t \rightarrow 0,
$$

with $\eta=O(1)$. In particular, via (2.5) and (2.7), we note that (2.8) represents a smoothed-out step approaching $u_{0}$ as $\eta \rightarrow \infty$ and 0 as $\eta \rightarrow-\infty$ with

$$
u(\eta, t) \sim \begin{cases}u_{0}-\hat{A} \eta^{-2 / 3} \exp \left(-3(-256 \gamma)^{-1 / 3} \eta^{4 / 3}\right) & \text { as } \eta \rightarrow \infty \\ \hat{A}(-\eta)^{-2 / 3} \exp \left(-3(-256 \gamma)^{-1 / 3}(-\eta)^{4 / 3}\right) & \text { as } \eta \rightarrow-\infty\end{cases}
$$

where $\hat{A}>0$ is a constant.

2.1.2. Region Ib: $x=O(1)>0$ As $\eta \rightarrow \infty$ we move into Ib, where $x=O(1)(>0)$ as $t \rightarrow 0$. The form of expansion (2.8) for $\eta \gg 1$ given by the first relation in (2.9) suggests that within Ib,

$$
u(x, t)=u_{0}-e^{-\phi(x, t)} \quad \text { as } t \rightarrow 0,
$$

with

$$
\phi(x, t)=\phi_{0}(x) t^{-1 / 3}+\phi_{1}(x) \ln t+\phi_{2}(x)+o(1),
$$

where $x=O(1)$ (but $\gg t^{1 / 4}$ ) as $t \rightarrow 0$ and $\phi_{0}(x)>0$. On substituting (2.10) and (2.11) into Equation (1.1) and solving at each order in turn, we find (after matching with (2.8) as $x \rightarrow 0^{+}$) that

$$
u(x, t)=u_{0}-\exp \left(-3(-256 \gamma)^{1 / 3} x^{4 / 3} t^{-1 / 3}+\frac{1}{6} \ln t+\left[\ln \hat{A}-\frac{2}{3} \ln x\right]+o(1)\right)
$$

as $t \rightarrow 0$, with $x=O(1)$.

2.1.3. Region Ic: $x=O(1)<0$ Finally, we consider the region where $x=$ $O(1)(<0)$ as $t \rightarrow 0$. The details in this case follow those given for $\mathbf{I b}$ albeit with some minor modification. Therefore in Ic it is sufficient to note that

$$
u(\hat{x}, t)=\exp \left(-3(-256 \gamma)^{1 / 3} x^{4 / 3} t^{-1 / 3}+\frac{1}{6} \ln t+\left[\ln \hat{A}-\frac{2}{3} \ln (-x)\right]+o(1)\right)
$$

as $t \rightarrow 0$.

The asymptotic structure as $t \rightarrow 0$ is now complete, with the expansions in regions Ic, Ia and Ib providing a uniform approximation to the solution of IVP as $t \rightarrow 0$. 
2.2. Asymptotic solution to IVP as $|\boldsymbol{x}| \rightarrow \infty$ for $\boldsymbol{t} \geq \boldsymbol{O}$ (1) The second stage of the analysis necessitates the investigation of the solution to IVP as $|x| \rightarrow \infty$ for $t=O$ (1). We first examine the structure of the solution to IVP as $x \rightarrow+\infty$ with $t=O(1)$. The form of expansion (2.12) of region Ib for $x \gg 1$ as $t \rightarrow 0$ suggests that in this region, which we label II,

$$
u(x, t)=u_{0}-e^{-\Theta(x, t)} \quad \text { as } x \rightarrow \infty,
$$

with

$$
\Theta(x, t)=\Theta_{0}(t) x^{4 / 3}+\Theta_{1}(t) x^{1 / 3}+\Theta_{2}(t) \ln x+\Theta_{3}(t)+o(1) \quad \text { as } x \rightarrow \infty
$$

and $t=O(1)$. On substituting (2.13) and (2.14) into Equation (1.1) and solving at each order in turn, we find (after matching with (2.12) as $t \rightarrow 0$ ) that

$$
\begin{aligned}
u(x, t)= & u_{0}-\exp \left[-\frac{3 t^{-1 / 3}}{4^{4 / 3}(-\gamma)^{1 / 3}} x^{4 / 3}+\frac{u_{0} t^{2 / 3}}{4^{1 / 3}(-\gamma)^{1 / 3}} x^{1 / 3}-\frac{2}{3} \ln x\right. \\
& \left.+\left[\frac{1}{6} \ln t+\ln \hat{A}\right]+o(1)\right]
\end{aligned}
$$

as $x \rightarrow \infty$ with $t=O(1)$. Expansion (2.15) will remain uniform for $t \gg 1$ provided that $x \gg t$, but it fails when $x \leq O(t)$.

We note the structure of the solution to IVP as $x \rightarrow-\infty$ with $t=O(1)$. The details in this case follow, subject to a few changes, those given above for region II and the results are summarized below. In region III,

$$
u(x, t)=\exp \left(-\frac{3 t^{-1 / 3}}{4^{4 / 3}(-\gamma)^{1 / 3}}(-x)^{4 / 3}-\frac{2}{3} \ln (-x)+\left[\frac{1}{6} \ln t+\ln \hat{A}\right]+o(1)\right)
$$

as $x \rightarrow-\infty$ with $t=O(1)$. As $t \rightarrow \infty$, the asymptotic expansion (2.16) in region III continues to remain uniform for $(-x) \gg t$. However, as already noted in the context of II, a nonuniformity develops when $x=O(t)$.

2.3. Asymptotic solution as $t \rightarrow \infty$ We are now able to fit the final piece into our puzzle by using the asymptotic expansions (2.15) and (2.16) for the solution in II and III to deduce the large-time solution for all $x$.

We begin by considering the asymptotic structure as $t \rightarrow \infty$ for $x>0$. To proceed it is helpful to consider a new region, which we denote by $\mathbf{I V}$, in which the scaled coordinate $y=x / t$ is $O(1)$ as $t \rightarrow \infty$. (The reader may find it helpful to refer to Figure 1 which shows the positioning in parameter space of $\mathbf{I V}$ and the additional zones introduced below.) Within IV, the form of (2.15) suggests that

$$
u(y, t)=u_{0}-e^{-t F(y, t)} \quad \text { as } t \rightarrow \infty,
$$

with

$$
F(y, t)=f_{0}(y)+f_{1}(y) t^{-1} \ln t+f_{2}(y) t^{-1}+o\left(t^{-1}\right) \quad \text { as } t \rightarrow \infty,
$$


with $y=O(1)$ and positive as $t \rightarrow \infty$ and $f_{0}(y)>0$. It is instructive to consider first the leading order problem in region IV. On substituting (2.17) and (2.18) into (1.1) we obtain the zeroth-order equation

$$
\gamma\left(f_{0}^{\prime}\right)^{4}+\left(y-u_{0}\right) f_{0}^{\prime}-f_{0}=0
$$

for $y>0$ together with the asymptotic behaviour

$$
f_{0}(y)=\frac{3}{4^{4 / 3}(-\gamma)^{1 / 3}} y^{4 / 3}-\frac{u_{0}}{4^{1 / 3}(-\gamma)^{1 / 3}} y^{1 / 3}+o\left(y^{1 / 3}\right) \quad \text { as } y \rightarrow \infty .
$$

Condition (2.20) arises by matching expansion (2.17) with $y \gg 1$ to the solution (2.15). Equation (2.19) admits a one-parameter family of linear solutions

$$
f_{0}(y)=\alpha\left(y-\left[(-\gamma) \alpha^{3}+u_{0}\right]\right), \quad y>0,
$$

for any $\alpha \in \mathbb{R}$ as well as the associated envelope solution

$$
f_{0}(y)=\frac{3}{4^{4 / 3}(-\gamma)^{1 / 3}}\left(y-u_{0}\right)^{4 / 3} .
$$

Combinations of (2.21) and (2.22) which remain continuous and differentiable are additional candidates as relevant solutions of (2.19) (these are the envelope touching solutions). If we recall that $f_{0}(y)>0$ then the condition (2.20) requires either

$$
f_{0}(y)= \begin{cases}3(-256 \gamma)^{-1 / 3}\left(y-u_{0}\right)^{4 / 3}, & y>u_{0}+4(-\gamma) \alpha^{3}, \\ \alpha\left(y-\left[(-\gamma) \alpha^{3}+u_{0}\right]\right), & u_{0}+(-\gamma) \alpha^{3}<y \leq u_{0}+4(-\gamma) \alpha^{3},\end{cases}
$$

where $\alpha>0$, or

$$
f_{0}(y)=3(-256 \gamma)^{-1 / 3}\left(y-u_{0}\right)^{4 / 3}, \quad y>u_{0} .
$$

We conclude that a nonuniformity occurs in expansion (2.17) as $y \rightarrow y_{c}^{+}\left(\geq u_{0}\right)$; if $f_{0}(y)$ satisfies (2.23) then $y_{c}=u_{0}+(-\gamma) \alpha^{3}$, but if $f_{0}(y)$ is given by (2.24) then $y_{c}=u_{0}$. More precise locations of the nonuniformity can be deduced by considering further terms in expansion (2.17), and this leads to the conclusion that nonuniformity occurs at $y=y_{c}+O(\Delta(t))$ as $t \rightarrow \infty$, where

$$
\Delta(t)= \begin{cases}t^{-1}, & \text { if } y_{c}=u_{0}+(-\gamma) \alpha^{3}, \\ t^{-3 / 4}, & \text { if } y_{c}=u_{0}\end{cases}
$$

We remark that although $f_{0}(y)$ and $f_{0}^{\prime}(y)$ are continuous over the range of definition of solution (2.23), the second derivative $f_{0}^{\prime \prime}(y)$ is discontinuous at the point at which the linear solution meets the envelope solution. This indicates that a thin transition region must surround the point of contact of the linear solution and the envelope in which these discontinuities in curvature are smoothed. We have two cases to examine, depending on whether $f_{0}(y)$ is given by (2.23) or (2.24). 
2.3.1. Transition zone structure if $f_{0}(y)$ is given by (2.23) In this case the nonuniformity occurs when $y=y_{c}+O\left(t^{-1}\right)$ so we introduce the transition region denoted TW. Here

$$
y=y_{c}+z t^{-1}
$$

where $y_{c}=u_{0}+(-\gamma) \alpha^{3}$ with $z=O(1)$ and $t \rightarrow \infty$. In TW the expansion (2.17) demonstrates that $u=O(1)$ as $t \rightarrow \infty$, and so we seek

$$
u(z, t)=U(z)+o(1), \quad z=O(1) \quad \text { as } t \rightarrow \infty .
$$

On substituting into Equation (1.1) we obtain the leading order problem

$$
\gamma U_{z z z z}-y_{c} U_{z}+U U_{z}=0, \quad-\infty<z<\infty,
$$

with $U(z)>0$ and

$$
\begin{array}{ll}
U(z) \rightarrow u_{0} & \text { as } z \rightarrow \infty, \\
U(z) \text { bounded } & \text { as } z \rightarrow-\infty .
\end{array}
$$

Condition (2.27) arises from matching (2.25) as $z \rightarrow \infty$ to (2.17) as $y \rightarrow y_{c}$.

On integrating Equation (2.26) and applying the boundary conditions as $z \rightarrow \infty$, we find that

$$
\gamma U_{z z z}-y_{c} U+\frac{1}{2} U^{2}=\frac{1}{2} u_{0}^{2}-y_{c} u_{0}
$$

and if $U(z) \rightarrow K$ as $z \rightarrow-\infty$ (where $K \neq u_{0}$ is some constant) we can replace condition (2.28) by

$$
U(z) \rightarrow 2 y_{c}-u_{0} \quad \text { as } z \rightarrow-\infty .
$$

We now demonstrate that there cannot be bounded solutions to (2.26)-(2.28). For on writing

$$
U=-\left(y_{c}-u_{0}\right) \Theta+y_{c}, \quad z=\frac{2^{1 / 3}(-\gamma)}{y_{c}-u_{0}} \xi,
$$

with $\Theta, \xi=O(1)$, boundary value problem (2.26), (2.27) and (2.29) becomes

$$
\begin{array}{ll}
\Theta_{\xi \xi \xi}=1-\Theta^{2}, & -\infty<\xi<\infty, \\
\Theta(\xi)>-1, & -\infty<\xi<\infty, \\
\Theta(\xi) \rightarrow \pm 1 & \text { as } \xi \rightarrow \pm \infty .
\end{array}
$$

We note that Equation (2.30) preserves its form under the transformation $\Theta \rightarrow-\Theta$, $\xi \rightarrow-\xi$ and therefore the solutions $\Theta$ either are anti-symmetric or occur in pairs. Without loss of generality we can choose the anti-symmetry point to be $\xi=0$. Clearly, there is no odd solution to (2.30)-(2.32) with $\Theta(0)=0$ and $\Theta( \pm \infty)= \pm 1$ since any such solution would have a discontinuity in the second derivative at $\xi=0$ $\left(\Theta^{\prime \prime}(0) \neq 0\right)$. Moreover, it has been established in [8] that (2.30) has a unique bounded solution, corresponding to a hetroclinic solution linking the fixed points $\Theta= \pm 1$, with $\Theta( \pm \infty)=\mp 1, \Theta(0)=\Theta_{\xi \xi}(0)=0$ and $\Theta_{\xi}(0)<0$, with all other solutions being unbounded and tending to infinity.

We conclude therefore that $f_{0}(y)$ cannot be given by (2.23) and so presumably satisfies (2.24) instead. 
2.3.2. Transition zone when $f_{0}(y)$ is given by (2.24) The substitution of (2.17) and (2.18) into Equation (1.1) gives, after solving order by order and matching to expansion (2.15) as $y \rightarrow \infty$, that

$$
u(y, t)=u_{0}-\exp \left(-\frac{3\left(y-u_{0}\right)^{4 / 3}}{4^{4 / 3}(-\gamma)^{1 / 3}} t-\frac{1}{2} \ln t+H(y)+o(1)\right) \quad \text { as } t \rightarrow \infty
$$

with $u_{0}+o(1)<y<\infty$. The function $H(y)$ cannot be tied down at this order, although matching to $(2.15)$ requires that

$$
H(y) \sim-\frac{2}{3} \ln y+\ln \hat{A} \quad \text { as } y \rightarrow \infty .
$$

As $y \rightarrow u_{0}^{+}$we move from region $\mathbf{I V}$ into the localized region, we which call $\mathbf{A}$.

An examination of (2.33) as $y \rightarrow u_{0}^{+}$reveals that in region $\mathbf{A}, y=u_{0}+O\left(t^{-3 / 4}\right)$ as $t \rightarrow \infty$. Within $\mathbf{A}$ we introduce the scaled coordinate $\zeta=\left(y-u_{0}\right) t^{3 / 4}$ and make the assumption (which we will later verify as consistent) that

$$
H(y) \sim \ln \left(y-u_{0}\right)^{1 / 3}+\ln D \quad \text { as } y \rightarrow u_{0}^{+}, \quad \text { where } D>0
$$

is some constant. Guided by (2.33) we expand

$$
u(\zeta, t)=u_{0}+G(\zeta) t^{-3 / 4}+o\left(t^{-3 / 4}\right) \quad \text { as } t \rightarrow \infty
$$

with $G(\zeta)<0$ and $\zeta=O(1)$. Substitution of (2.34) into Equation (1.1) gives

$$
\gamma G_{\zeta \zeta \zeta \zeta}+G G_{\zeta}-\frac{\zeta}{4} G_{\zeta}-\frac{3}{4} G=0
$$

which is to be solved subject to matching with region IV as $\zeta \rightarrow \infty$; hence

$$
G(\zeta) \sim-D \zeta^{1 / 3} \exp \left(-3(-256 \gamma)^{-1 / 3} \zeta^{-4 / 3}\right) \quad \text { as } \zeta \rightarrow \infty .
$$

Finally, for $u$ to be bounded as $t \rightarrow \infty$ when $y=u_{0}+O(1)$, we require

$$
\zeta^{-1} G(\zeta) \text { bounded as } \zeta \rightarrow-\infty \text {. }
$$

We note that $G(\zeta)=\zeta$ is a simple solution of (2.35), and further that if $G(\zeta)$ is a solution of (2.35) then so is $-G(-\zeta)$.

The leading order problem is now complete, and is governed by (2.35) and (2.37). This boundary-value problem is both nonlinear and nonautonomous, although, apart from $D>0$, it is parameter-free (the nature of the solution is qualitatively independent of $\gamma<0)$.

Equation (2.35) was investigated numerically by applying asymptotic conditions (2.36) and then shooting towards $\zeta=-\infty$. Experiments proved that there is a crucial value $D=D^{*}(\gamma)$ for which a solution which satisfies (2.37) exists. 


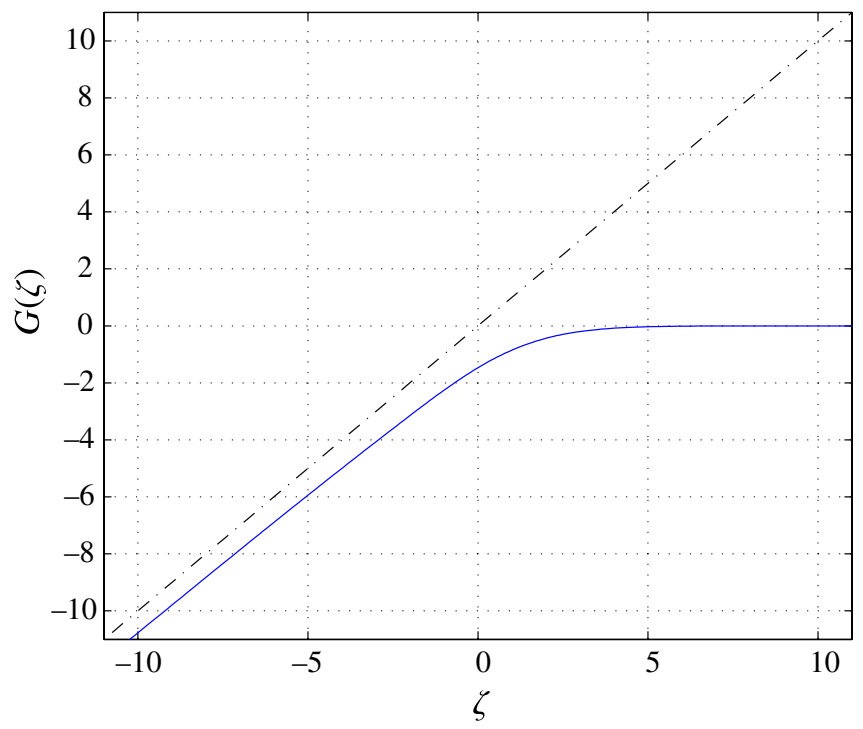

FIGURE 1. The solid line represents the numerically computed solution $G^{*}(\zeta)$ to $(2.35)$ and (2.36) when $\gamma=-1$, while the dashed line represents the solution $G(\zeta)=\zeta$.

If either $D<D^{*}$ or $D>D^{*}$ then the solution of (2.35) which satisfies the asymptotic condition (2.36) blows up at a finite point $\zeta=\zeta_{0}(D)$ according to

$$
G(\zeta)=O\left[\left(\zeta-\zeta_{0}\right)^{-3}\right] \quad \text { as } \zeta \rightarrow \zeta_{0}
$$

This suggests that the boundary-value problem (2.35)-(2.37) has a unique solution for $D=D^{*}$, say $G=G^{*}(\zeta)$; this function is shown in Figure $1 . G^{*}(\zeta)$ is monotonic increasing so that, in particular, $G^{*}(\zeta)<0$ for all $\zeta$ and it can be shown that

$$
G^{*}(\zeta) \sim \zeta+O\left((-\zeta)^{-1 / 3}\right) \quad \text { as } \zeta \rightarrow-\infty .
$$

As $\zeta \rightarrow-\infty$ we leave the localized region $\mathbf{A}$ and enter a new regime in which $y<u_{0}-o(1)$. If this zone is designated region $\mathbf{V}$, the form of solution here follows from (2.34) and (2.38). In particular,

$$
u(y, t)=\widehat{F}(y)+O\left(t^{-1}\right) \quad \text { as } t \rightarrow \infty
$$

with $\hat{F}(y)>0$ and where $y<u_{0}-O\left(t^{-3 / 4}\right)$ as $t \rightarrow \infty$. The substitution of (2.39) into Equation (1.1) leads to $\widehat{F}_{y}(\widehat{F}-y)=0$, for $y<u_{0}$. Moreover, we need $\widehat{F}(y)>0$ for $y<u_{0}$ and expansion (2.39) will match to (2.34) for $(-\zeta) \gg 1$ only if $\widehat{F}(y) \sim y$ as $y \rightarrow u_{0}^{-}$. This then forms an easy problem with solution simply $\widehat{F}(y)=y$ for $0<y<u_{0}$. The upshot is that in region $\mathbf{V}$ we have

$$
u(y, t)=y+O\left(t^{-1}\right)
$$

as $t \rightarrow \infty$ where $0<y<u_{0}$. 
Expansion (2.40) clearly becomes nonuniform as $y \rightarrow 0^{+}$and this points to the need for a further localized region, region $\mathbf{B}$, in which $y=o(1)$ and $u(y, t)=o(1)$ as $t \rightarrow \infty$.

2.3.3. The localized region $\boldsymbol{B}$ To examine $\mathbf{B}$, we introduce the scaled coordinate $\xi=y t^{\sigma}=O(1)$ as $t \rightarrow \infty$, where $\sigma>0$, and look for an expansion of the form (as suggested by (2.40))

$$
u(\xi, t)=\bar{u}(\xi) t^{-\sigma}+o\left(t^{-\sigma}\right),
$$

as $t \rightarrow \infty$ with $\bar{u}=O(1)$. On substitution of expansion (2.41) into Equation (1.1) we find that $\sigma=3 / 4$ and then

$$
\gamma \bar{u}_{\xi \xi \xi \xi}+\bar{u} \bar{u}_{\xi}-\frac{\xi}{4} \bar{u}_{\xi}-\frac{3}{4} \bar{u}=0
$$

which is to be solved subject to matching with region $\mathbf{V}$, that is,

$$
\bar{u}(\xi) \sim \xi \quad \text { as } \xi \rightarrow \infty,
$$

together with the need for $\xi^{-1} \bar{u}(\xi)$ to be bounded as $\xi \rightarrow-\infty$. This problem is just a repeat of the key equation (2.35) that governed the solution structure in A. Since we note that if $G(\zeta)$ is a solution of (2.35) then so is $-G(-\zeta)$, we can be assured of the existence of a positive, monotone increasing solution to (2.42) that satisfies the matching condition (2.43) and which has the asymptotic form

$$
\bar{u}(\xi) \sim D^{*}(-\xi)^{1 / 3} \exp \left(-3(-256 \gamma)^{-1 / 3}(-\xi)^{4 / 3}\right) \quad \text { as } \xi \rightarrow-\infty .
$$

Our task is now nearly complete. All that remains is to develop the asymptotic structure as $t \rightarrow \infty$ for $x<0$. Earlier we derived the form of the solution (2.16) corresponding to $x \rightarrow-\infty$ with $t=O(1)$ (region III). It was noted that this asymptotic expression remains uniform for $(-x) \gg t$ but that a disordering occurs when $(-x)=O(t)$. To resolve this nonuniformity we must consider one last zone, region VI, in which the coordinate $y=x / t$ is $O(1)$ as $t \rightarrow \infty$. The structure of (2.16) suggests that within VI,

$$
u(y, t)=e^{-t \phi(y, t)}
$$

where

$$
\phi(y, t)=\phi_{0}(y)+\phi_{1}(y) t^{-1} \ln t+\phi_{2}(y) t^{-1}+o\left(t^{-1}\right) \quad \text { as } t \rightarrow \infty,
$$

with $y=O(1)(<0)$ and $\phi_{0}(y)>0$. The leading order problem in region $\mathbf{V I}$ is then

$$
\gamma\left(\phi_{0}^{\prime}\right)^{4}+y \phi_{0}^{\prime}-\phi_{0}=0, \quad y<0
$$

with $\phi_{0}(y)>0$ and matching with regions III and $\mathbf{B}$ requires

$$
\phi_{0}(y) \sim 3(-256 \gamma)^{-1 / 3}(-y)^{4 / 3} \equiv \Phi_{0}(y)
$$




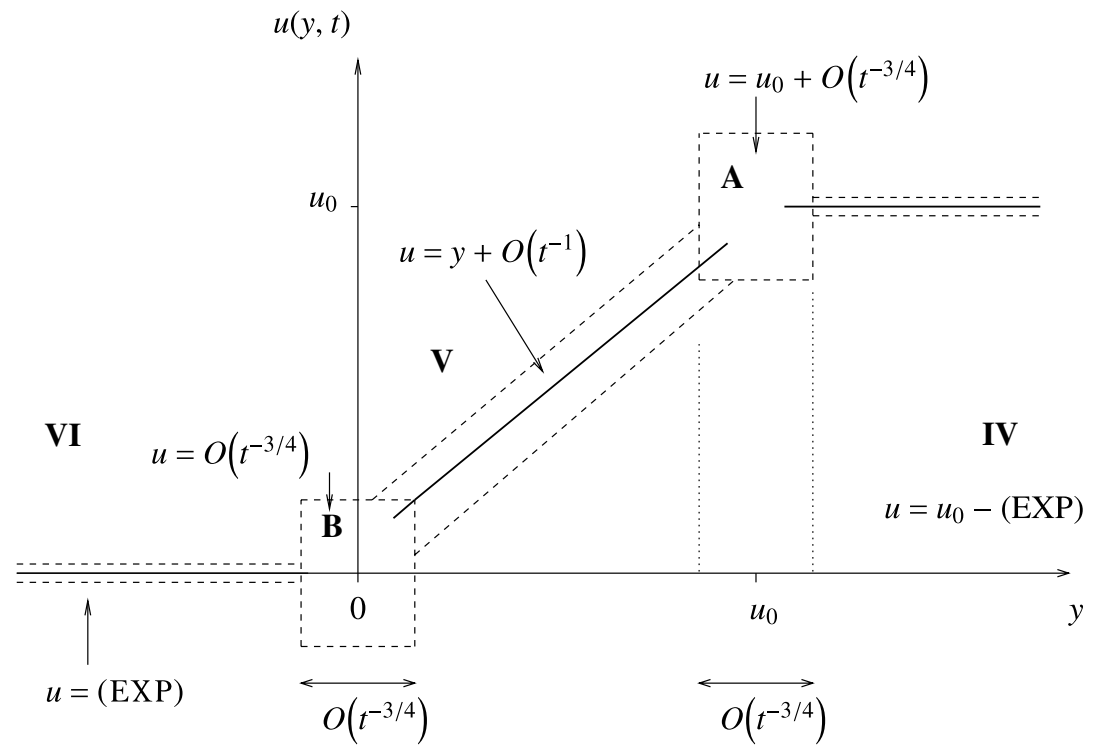

FIGURE 2. A schematic representation of the asymptotic structure of $u(y, t)$ in the $(y, u)$ plane, as $t \rightarrow \infty$. Here (EXP) denotes terms exponentially small in $t$ as $t \rightarrow \infty$.

both as $y \rightarrow 0^{-}$and $y \rightarrow-\infty$. The solution then is simply $\phi_{0} \equiv \Phi_{0}(y)$ for all $y<0$. The substitution of (2.44) and (2.45) into Equation (1.1) gives that

$$
u(y, t)=\exp \left(-\frac{3(-y)^{4 / 3}}{4^{4 / 3}(-\gamma)^{1 / 3}} t-\frac{1}{2} \ln t+\hat{H}(y)+o(1)\right) \quad \text { as } t \rightarrow \infty
$$

where $-\infty<y<0-o(1)$. The function $\hat{H}(y)$ is indeterminate at this order but matching to region $\mathbf{B}$ (as $y \rightarrow 0^{-}$) and to the far field (as $y \rightarrow-\infty$ ) implies that

$$
\hat{H}(y) \sim \begin{cases}-\frac{2}{3} \ln (-y)+\ln \hat{A}, & \text { as } y \rightarrow-\infty, \\ \frac{1}{3} \ln (-y)+\ln D, & \text { as } y \rightarrow 0^{-} .\end{cases}
$$

The asymptotic structure to the solution of IVP as $t \rightarrow \infty$ is now complete. A uniform approximation has been derived through regions III, VI, B, V, A, IV and II. A sketch of the overall asymptotic structure of $u(x, t)$ as $t \rightarrow \infty$ is given in Figure 2 .

\section{Discussion}

In this paper we have obtained, via the method of matched asymptotic coordinate expansions, the large- $t$ solution to IVP who principal feature is the formation of an expansion wave. This large- $t$ structure has been derived by a careful combination of the asymptotic structures as $t \rightarrow 0$ for $|x|<\infty$ and of the $|x| \rightarrow \infty$ form appropriate to $t \geq O(1)$. The large- $t$ structure consists of five main regions, shown in terms of the coordinate $y$ (where $y=x / t$ ) in Figure 2 . 
Regions IV and VI allow the transfer of information from the far field $|y| \gg 1$ to the near field $y=O(1)$. At leading order in region IV the solution to IVP is $O(1)$ and is given by the constant value $u_{0}$ (the value of $u$ ahead of the expansion wave). The correction to this value is only exponentially small in $t$ as $t \rightarrow \infty$. As $y \rightarrow u_{0}^{+}$ so the solution in IV becomes nonuniform and this is resolved within a localized region $\mathbf{A}$ which is centred around $y=u_{0}$. This localized region then connects to $\mathbf{V}$ when $0<y<u_{0}$ as $t \rightarrow \infty$, where $u=O(1)$ and is given by the expansion wave at leading order. In VI, where $y<0$, the solution is exponentially small in $t$. As $y \rightarrow 0^{-}$ the relevant expansion again becomes nonuniform and it is only within $\mathbf{B}$ that this disordering can be resolved.

We reiterate that the initial value problem solved here is ill-posed inasmuch as the part of the profile where $u=0$ is linearly unstable. In any practical application we would expect $u(x, 0) \neq 0$ for all $x$, but the basic ideas of tracking the development of an initial profile containing a discontinuous expansive step remain unaltered. Moreover, in most fourth-order diffusion problems $\gamma>0$ but, once again, the fundamental concepts of exploring the long-time development of such a system are well illustrated by our considerations. In summary, then, this work, although very much a first study of IVP, opens up a number of avenues for possible extension. First, given that previous studies have shown how the evolution of reaction-diffusion equations is crucially dependent on the nature of the initial profile, it would be of interest to see how the predictions made above need to be modified should $u(x, 0)$ be a continuous function with compact support. There is also clearly the need for further work on advanced numerical methods in order to simulate our model equation accurately through all times. Finally, the outcomes of these studies will inform the application of matched asymptotic expansions to the physically significant KuramotoSivashinsky equation. We hope to explore some of these issues in future work.

\section{References}

[1] P. Broadbridge and P. Tritscher, "An integrable fourth-order nonlinear evolution equation applied to thermal grooving of metal surfaces", IMA J. Appl. Math. 53 (1994) 249-265.

[2] D. A. Caughey and M. M. Hafez (eds), Frontiers of computational fluid dynamics (World Scientific, Singapore, 2006).

[3] V. Galaktionov, "On higher-order viscosity approximations of odd-order nonlinear PDEs", J. Engng. Math. 60 (2008) 173-208.

[4] E. J. Hinch, Perturbation methods (Cambridge University Press, Cambridge, 1991).

[5] Y. N. Kyrychko, M. V. Bartuccelli and K. B. Blyuss, "Persistence of travelling wave solutions of a fourth-order diffusion system”, J. Comput. Appl. Math. 176 (2005) 433-443.

[6] P. A. Lagerstrom and R. G. Casten, "Basic concepts underlying singular perturbation techniques", SIAM Rev. 14 (1972) 63-120.

[7] J. A. Leach and D. J. Needham, Matched asymptotic expansions in reaction-diffusion theory (Springer, London, 2003).

[8] C. K. McCord, "Uniqueness of connecting orbits in the equation $y^{(3)}=y^{2}-1$ ", J. Math. Anal. Appl. 114 (1986) 584-592.

[9] A. H. Nayfeh, Introduction to perturbation techniques (Wiley, New York, 1981).

[10] M. Van Dyke, Perturbation methods in fluid dynamics (Parabolic Press, Stanford, 1975). 